\title{
MANIFESTACIONES DE LA DINÁMICA FAMILIAR EN FACTORES DE RIESGO SOCIOCULTURALES QUE INCIDEN EN EL CONSUMO DE ALCOHOL EN JÓVENES DE 12 A 18 AÑOS EN TIEMPO DE COVID 19 \\ MANIFESTATIONS OF FAMILY DYNAMICS IN SOCIOCULTURAL RISK FACTORS THAT AFFECT THE ALCOHOL CONSUMPTION IN YOUNG PEOPLE OF 12 TO 18 YEARS IN COVID-19 TIME
}

\section{Clara Judith Brito Carrillo ${ }^{1}$}

\section{Carmenza Leonor Mendoza Cataño}

Angélica María Suarez Brito ${ }^{3}$

Universidad de La Guajira

\footnotetext{
1 Mcs. Desarrollo y Gestión de empresas sociales Gerencia Social. Trabajadora Social, Docente Universidad de la guajira. Investigador asociado, líder Grupo de Investigación: Encuentro con la investigación Categorizado en A1. Email.clarabrito@ uniguajira.edu.co . https://orcid.org/0000-0001-8788-7326 la Guajira. Investigador asociado. Art. Inclusión Social En Prevención De Conductas Adictivas En Ambientes Escolares: Desafíos Del Trabajador Social. 2016. escenarios@trabajosocial.unlp.edu.arl. Carmenza Leonor Mendoza CataÃño cmendoza@uniguajira.edu.co

$2 \quad$ PHD En Gerencia de la Educación Superior. Doctora en Ciencias Gerencial Magister en Gerencia de Recursos Humanos. Especialista en Pedagogía para el aprendizaje Autónomo Psicóloga

$3 \quad$ Psicóloga. especialista en psicología clínica autoeficacia personal. Docente Catedrática de la Universidad de la Guajira. Email. angelicasuarez@uniguajira.edu.co https://orcid.org/0000-0002-6577-7393
} 


\section{RESUMEN}

El presente artículo tuvo como objetivo. Identificar los factores de riesgo socioculturales que inciden en el consumo de alcohol en jóvenes de 12 a 18 años de edad en la institución educativa Pablo VI en el municipio de Barrancas La Guajira, desde diferentes perspectivas se caracterizó por su proceso metodológicamente, implícito en la investigación de tipo descriptiva, enfoque cuantitativo, diseño no experimental, transeccional y de campo, la población fue 30 estudiantes. Los resultados evidencian situaciones problematizadas que requieren de acciones articuladas para buscar alternativas dada la relevancia para la salud mental. Se destaca la Guía de Intervención mhGAP, 2010, de acuerdo con la cual es necesario que se "Promueva un cambio en el entorno del adolescente en lugar de enfocarse directamente en el adolescente como el problema. Por ejemplo, promueva la participación en la escuela o el trabajo y en actividades después de clase / trabajo que ocupen el tiempo del adolescente, y promueva la participación en actividades de grupo que faciliten la adquisición de habilidades y la contribución a sus comunidades. Es importante que los adolescentes se involucren en actividades que les interesen" (p.63)

PALABRAS CLAVE: Consumo de alcohol, dinámica familiar, factores de riesgo $\mathrm{y}$ socioculturales,

\section{ABSTRACT}

This article was intended. Identify the sociocultural risk factors that influence alcohol consumption in young people from 12 to 18 years of age in the Pablo $\mathrm{VI}$ educational institution in the municipality of Barrancas La Guajira. From different perspectives, it was characterized by its methodological process, implicit in the research. descriptive, quantitative approach, non-experimental, cross-sectional and field design, the population was 25 students.
Whose results show problematic situations that require articulated actions to look for alternatives given the relevance for mental health, likewise, the theoretical bases, guaranteed the integrated strengthening of the study objective, in view of the above, the mhGAP Intervention Guide stands out, 2010, it is necessary to "Promote a change in the adolescent's environment instead of focusing directly on the adolescent as the problem. For example, promote participation in school or work and in after-school / work activities that occupy the adolescent's time, and promote participation in group activities that facilitate the acquisition of skills and contribution to their communities. It is important that adolescents get involved in activities that interest them" (p.63)

KEY WORDS: Alcohol consumption, family dynamics, risk and sociocultural factors,

\section{INTRODUCCIÓN}

La organización Mundial de la salud (OMS), y la Organización Panamericana de la salud (OPS), 2010, puntualizan "Recomiende a los padres limitar las conductas propias que puedan estar contribuyendo al uso de sustancias de sus hijos, incluyendo la compra o suministro de alcohol o la provisión de dinero que se gasta en el uso de sustancias, teniendo en cuenta la posible influencia que su propio consumo de alcohol y sustancias puede tener en sus hijos"

Dentro de este contexto, esta investigación posee una connotación de gran relevancia y pertenencia, porque visibiliza que el consumo al alcohol en jóvenes y adolescentes es inminente debido a la influencia de efectos culturales, si se tiene en cuenta que se inician a temprana edad al interior de las familias, como factor que predispone a esas conductas propias de la región, donde no se toman las medidas correctivas necesarias en cuanto a sus ingestas.

En este orden de ideas, se puede confirmar, que esta problemática posee unas dimensiones 
sociofamiliares, culturales, económicas y políticas, las cuales de una u otra forma inciden en que los jóvenes y adolescentes decidan a temprana edad que el consumo de alcohol es una alternativa de solución frente a las situaciones conflictivas que enfrentan en la relación directa familias, instituciones educativas y por ende la comunidad

\section{Esquema 1 Manifestaciones de la dinámica familiar que Inciden en el Consumo de Alcohol}

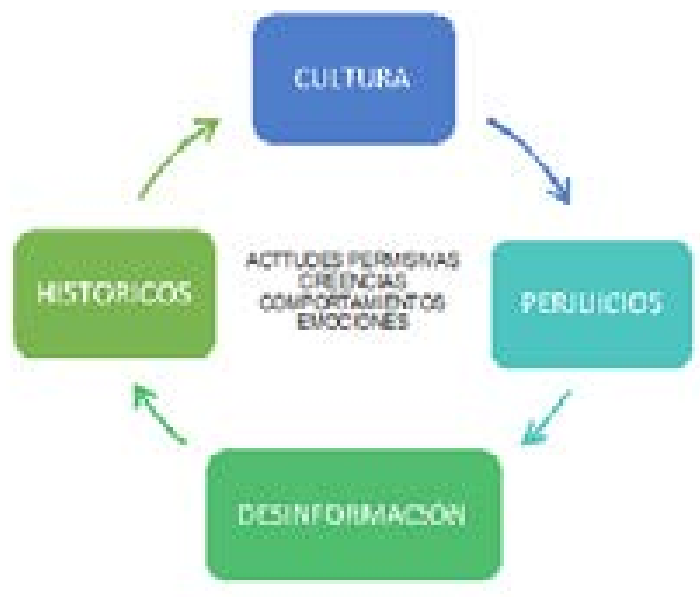

Elaboración propia Brito Carrillo, Mendoza Cataño \& Suarez Brito. 2020

En síntesis, el alcohol ha jugado un importante papel en la historia de la humanidad. En la mayoría de las sociedades, beber es esencialmente un acto social y está muy arraigado en un contexto de valores, actitudes y tradiciones. Las actitudes hacia el alcohol tienen una gran importancia, pues influyen poderosamente en el consumo de bebidas alcohólicas. El cruce de culturas indica que las actitudes de la gente hacia el alcohol varían de forma sustancial (Heath, 1988,1995).

Dentro de este contexto, se visibilizan las diferentes manifestaciones de la dinámica familiar en factores de riesgo socioculturales que inciden en el consumo de alcohol en jóvenes de 12 a 18 años en tiempo de covid-19, lo cual agudiza la situación debido a la flexibilidad, desesperanza, violencia intrafamiliar, abandono, estudiantes que no conviven con sus padres biológicos, y en los actuales momentos el temor al contagio porque no respetan las normas de confinamiento social exigidas para la prevención de la pandemia mundial que se ha convertido en una crisis global con grandes repercusiones para la vida familiar y social, porque existe un rompimiento de valores tradicionales, que por años se han transmitido de generación en generación en cuanto a las costumbres culturales del municipio de Barrancas y por ende ene I departamento de La Guajira.

No obstante, los padres de familias quienes de una $u$ otras formas han incidido negativamente para que se susciten situaciones conflictivas al interior del hogar, lo cual se justifica que el alcohol es propio de la cultura de la región no los exonera de comportamiento inadecuado que presentan sus hijos. Por otra parte cabe destacar que existe desconocimiento de la estrategia nacional pactos por la vida, de igual manera no se establecen programas continuos entre los actores sociales Empresas Social del Estado Hospital Nuestra Señora del Pilar, Secretaria de Educación y Salud, denotándose que la administración municipal vigencia 2018 / 2019, no se lideran acciones de prevención frente al consumo de alcohol en el ámbito escolar y no se promocional estilos de vida saludables, que fortalezcan la utilización del tiempo libre productivo de la comunidad estudiantil.

\section{DESARROLLO}

Al respecto, se puntualizan que, a nivel nacional, a han dictado medidas para la prevención del consumo de alcohol en menores y adolescentes, en otras palabras, se puede visibilizar

La implementación de la política de alcohol en Colombia, está articulada a la Estrategia Mundial de reducción del uso nocivo de alcohol, aprobada por la Asamblea Mundial de la Salud 
en el año 2010, donde se reconoce que el uso nocivo de alcohol tiene graves repercusiones a la salud pública y es considerado uno de los principales factores de riesgo a nivel mundial.

La estrategia nacional se articula a la estrategia mundial en las siguientes diez esferas: 1. liderazgo, concientización y compromiso. 2 . respuesta de los servicios de salud. 3. acción comunitaria. 4. políticas y medidas contra la conducción bajo los efectos del alcohol. 5 . disponibilidad de alcohol. 6. mercadeo de bebidas alcohólicas. 7. políticas de precios. 8 . mitigación de las consecuencias negativas del consumo de alcohol y la intoxicación etílica. 9. reducción del impacto en la salud pública del alcohol ilícito y el alcohol de producción informal. 10. seguimiento y vigilancia. (Ministerio de salud 2015)

En vista de lo anterior se denotan La familia es el primer ámbito social del ser humano, donde aprende los primeros valores, principios y nociones de la vida. Es un grupo social con una base afectiva y formativa, donde conviven personas unidas por lazos de consanguinidad y amor mutuo. Este grupo humano es la célula básica de la sociedad, puesto que las civilizaciones nacieron con alguna forma de organización familiar, que fue variando a través de los tiempos en la humanidad. (Fishman, 2015)

Así mismo, la familia es un contexto complejo e integrador, que participa en la dinámica interrelacionar de los sistemas biológico, psicosocial y ecológico, dentro del cual existen pautas, normas, deberes y derechos que contribuyen a una sana convivencia, es decir, la coexistencia de un carácter pacífico, armonioso y sereno, alejándose totalmente de las disputas, riñas o discusiones. "(Tuvilla, 2004)

\section{GRUPOS MÁS VULNERABLES}

Los grupos más vulnerables al consumo respecto de la edad van desde los 15 hasta los 35 años, especialmente, con el agravante de que los bebedores jóvenes duplican el riesgo de mantener su conducta de consumo abusiva durante la edad adulta y la vejez (OMS, 2010a). En las Américas, el promedio de consumo entre los adultos es perjudicial, ya que en los jóvenes el consumo abusivo tiene un carácter más episódico y su inicio se da a cada vez menor edad (OPS, 2007). Para 2012, el 61,7\% de la población mundial mayor de 15 años había tomado alguna bebida alcohólica, especialmente entre los hombres, y en los jóvenes cerca del $16 \%$ presentaba alto consumo episódico (OMS, 2014)

\section{INFLUENCIA DE AMIGOS}

Influencia del grupo de amigos y de los pares ha sido vista como un factor de riesgo importante para el consumo, y esto no es exclusivo de los adolescentes, pues las personas mayores también buscan identificarse con su grupo escolar y laboral de homólogos, por cuanto con ellos comparten la mayor parte del tiempo (Alfonso, Huedo-Medina y Espada, 2009; Previte, Fry, Drennan y Hasan, 2015). Por ello, son susceptibles a ceder ante la presión directa e indirecta en torno al consumo de alcohol ejercida por los pares (Andrade, Yepes y Sabogal, 2013; Londoño y Valencia, 2008, 2010); o sencillamente, las creencias acerca de la importancia de beber en distintos momentos y ambientes relacionados con intercambio social y diversión hacen que no quieran resistir la invitación a beber que le hace su grupo de referencia (Londoño, Valencia, Sánchez y León, 2007). 


\section{PREVENCIÓN DE CONSUMO DE ALCOHOL EN JÓVENES}

Se asocia las palabras "diversión", "disfrutar", "celebración", "alegría", "pasarla bien" o "amigos" con las bebidas alcohólicas, entonces las estrategias de publicidad de la industria del alcohol han dado resultado. Es más, es posible que algunas fiestas culturales o los deportes más populares, los torneos, equipos o los deportistas que los practican ya estén relacionados con el sello de alguna bebida alcohólica y sea muy difícil pensar en éstos sin recordar la marca o el eslogan de sus comerciales. (Ministerio de salud. 2015, p.27)

\section{- $\quad$ REDUCCIÓN DE RIESGOS}

El concepto plantea minimizar las condiciones de riesgo asociadas al consumo de drogas, estableciendo un contacto directo y permanente con los consumidores en las mismas comunidades locales en donde se da el fenómeno. Reducir riesgos significa trabajar en la cotidianidad y crear espacios de bajo umbral donde las exigencias para ingresar sean mínimas. (Modelo de inclusión - 2007). República de Colombia. Ministerio de Protección Social y la Oficina de las Naciones Unidas contra la Droga y el Delito (2010. P. 23)

En otras palabras, las acciones no son coherentes con los lineamientos existentes a nivel nacional donde se han establecido los acuerdos a través del Ministerio de Salud y Protección Social, 2013 a través de la estrategia...

"Pactos por la Vida" hace referencia a la búsqueda de acuerdos entre diversas instancias sociales que tienen un papel sustancial en la cadena de eventos conducentes a la producción, comercialización, y consumo de bebidas alcohólicas, con la finalidad de que ese consumo se dé en condiciones que no atenten contra la vida de los consumidores, enfatizando en la necesidad de prevenir y de mitigar la multitud de consecuencias negativas potenciales que el consumo de alcohol representa para la salud y para el bienestar de los consumidores y de las personas relacionadas en su entorno social, consecuencias que configuran un problema de salud pública. (I)

\section{RESULTADO DE INVESTIGACIÓN}

La metodología implícita en investigación, contempla el tipo o tipos de investigación, las técnicas y los procedimientos que serán utilizados para llevar a cabo la indagación. Es el "cómo" se realizará el estudio para responder al problema planteado" (ARIAS ODON. Fidias (1999). Incidencia cultural del consumo de alcohol en jóvenes de 12 a 18 años de edad en la institución educativa pablo $\mathrm{VI}$ en el municipio de Barrancas La Guajira. De todos modos, se establecen en los capítulos del marco metodológico los elementos relevantes de la investigación. Así mismo se exponen los aspectos descritos al tipo de investigación realizada, el diseño, la población y muestra sobre la cual se trabajó la investigación, dentro de este contexto aplicabilidad de las técnicas e instrumento de recopilación de los datos. Por su parte se señala, la confiabilidad y validez del instrumento, el análisis de los datos recolectados y el procedimiento que fue desarrollado para llevar a cabo la investigación

\section{ENFOQUE DE LA INVESTIGACIÓN}

Esta investigación es de modelo cualicuantico, porque proporciona una gran compresión de las razones y motivaciones subyacentes de las personas. El enfoque cuantitativo usa la recolección de datos para probar hipótesis con base a la medición numérica y el análisis y estadísticos para establecer patrones de comportamiento y probar teorías 


\section{TIPO DE INVESTIGACIÓN}

Esta investigación será de tipo descriptivo, debido a que busca identificar la percepción que tienen las personas sobre el objeto del estudio

\section{DISCUSIÓN DE RESULTADOS}

En este sentido, al analizar las incidencias culturales del consumo de alcohol en jóvenes de 12 a 18 años de edad en la institución educativa Pablo VI en el municipio de Barrancas La Guajira, se entrevé que existe un consumo de alcohol aceptado culturalmente, el cual no es observado como una problemática de salud, puesto que las repercusiones a futuro poseen unas implicaciones lesivas para la salud de los jóvenes ya adolescentes, es por ello, que desde la Secretaria de Educación municipal, se requiere del fortalecimiento de programas de prevención y mitigación del mismo, a través de un abordaje integral de las situaciones conflictivas existentes en la Institución educativa, familia y por ende en la comunidad

\section{Grafica 1 ¿la institución educativa PAULO} VI municipio de Barrancas La Guajira, los factores culturales influyen para que los estudiantes consuman alcohol?
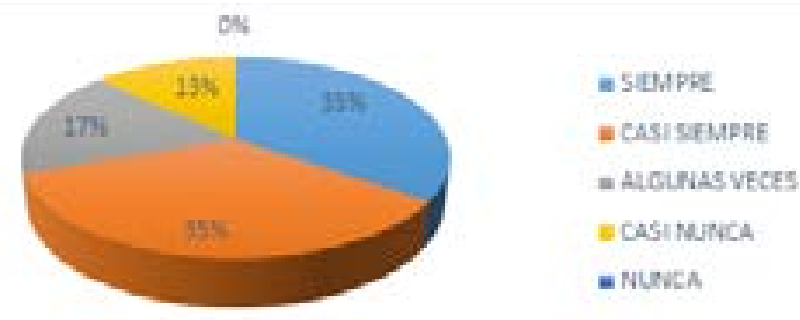

Fuente: investigadores. Brito Carrillo, Mendoza Cataño \& Suarez Brito. 2020

En la gráfica se observa, que en la Institución educativa Paulo VI localizado en el Municipio de Barrancas, La Guajira, si existe consumo de alcohol, lo cual posee componentes culturales denotándose que los estudiantes expresan la existencia de factores de riesgo, es por ello que se requiere acciones articuladas para prevenir las secuelas del consumo de alcohol. En sectores vulnerables de la sociedad barranquera

Grafica 2 La administración municipal de Barrancas La Guajira realiza programas institucionales a través de la estrategia pactos por la vida para la prevención del consumo de alcohol

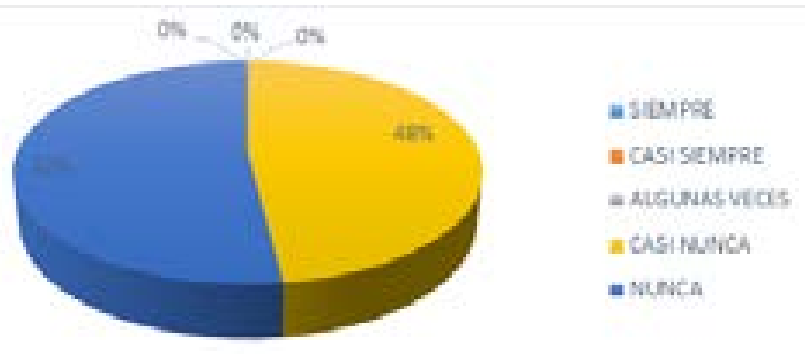

Fuente: investigadores. Brito Carrillo, Mendoza Cataño \& Suarez Brito. 2020

Es Preciso Resaltar, que la comunidad educativa Paulo VI, es consiente que la Administración Municipal de Barrancas La Guajira, No realiza programas institucionales a través de la estrategia pactos por la vida para la prevención del consumo de alcohol, denotándose un $52 \%$ de Nunca y un $48 \%$ casi nunca, lo cual es representativo por las situaciones problematizadas debido a la no aplicabilidad de la política nacional de drogas, y a las exigencias de la ley 1616 de febrero de 2013.

Grafica 3 considera usted que los padres de familias deben involucrarse en las escuelas para padres para adquirir conocimientos en la prevención del consumo de alcohol

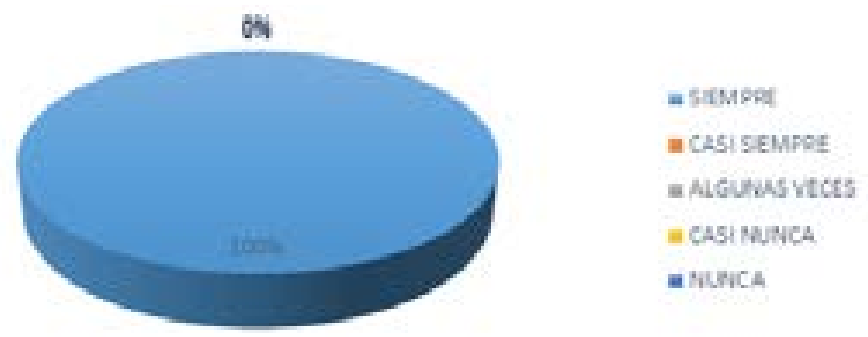

Fuente: investigadores. Brito Carrillo, Mendoza Cataño \& Suarez Brito. 2020 
Como es de esperarse ¡las afirmaciones de los estudiantes, las cuales son asertivas en un $100 \%$, cuando son sus mismos hijos, quienes consideran que los padres de familias deben involucrarse en las escuelas para padres para adquirir conocimientos en la prevención del consumo de alcohol, es por ello, que se hace una llamado a los padres de Familias de manera general para que conjuntamente con la Institución Educativa articulen acciones preventivas frente al consumo de alcohol y otras situaciones conexas

\section{Grafica 4 Problemáticas sociales y familiares existentes en el Municipio de Barrancas que se evidencian en la institución educativa Paulo VI son productos de los casos del consumo de alcohol existentes en los jóvenes y adolescentes}

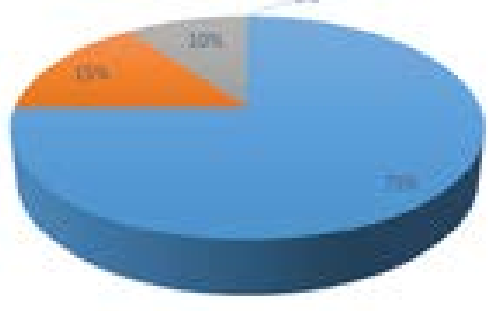

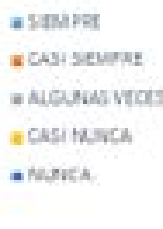

Fuente: investigadores, Brito Carrillo, Mendoza Cataño \& Suarez Brito. 2020

Se evidencian que la problemáticas sociales y familiares existentes en el Municipio de Barrancas La Guajira, son productos del consumo de alcohol en los sectores vulnerables de jóvenes y adolescentes, es por ello, que es necesario replantear acciones integradoras porque el $75 \%$ de los estudiantes expresan que el surgimiento multiplicidad de problemáticas sociales al interior del hogar es producto del consumo de alcohol.

Indiscutiblemente, se requiere de generar espacios de reflexión y análisis, donde se propicien acciones integradoras para disminuir y/o erradicar mediante la mitigación y prevención, las secuelas producidas por esta problemática social es por ello, que se destaca Lisa Kohn, 2015, vocera del proyecto "No más", "contra la violencia doméstica y el abuso sexual", afirma que al principio, muchas mujeres ni se dan cuenta de que son víctimas de abuso, sino que creen que todo se debe a factores como el estrés, problemas en el trabajo, alcohol, drogas, es por ello, que se requiere de la consolidación de un equipo de trabajo interdisciplinario para la implementación de estrategia donde se involucre a la comunidad para fomentar estilo de vida saludables debido a los trastornos que se presentan por la violencia de parejas en la cual tanto hombres como mujeres son víctimas directas de esta situación al interior de la familia

\section{CONCLUSIONES}

En la Institución educativa Paulo VI del municipio de Barrancas La Guajira, existen una multiplicidad factores de riesgo, entre ellos, unos arraigados patrones culturales a nivel familiar, social y comunitarios, lo cual incide para que se presente esta situación de riesgo que inestabiliza el comportamiento de la comunidad estudiantil, por otra parte es evidente, que escasamente se realicen acciones preventivas tales como acciones socioeducativa, es por ello, que los estudiantes establecen juicios de valores despectivamente con relación a los resultados de las charlas en su relación con el alcohol

Es evidente, la necesidad expresada por los estudiantes la aplicabilidad de establecer estrategias para la reducción de las diferentes manifestaciones del consumo abusivo de alcohol, las cuales son muy variadas y algunas implican riesgos debido a la alta ingesta de alcohol, el cual se ha extendido en la población de jóvenes y adolescentes.es por ello, que surge la necesidad de consolidar estrategias para la reducción de los riesgos los cuales alteran el comportamiento de los adolescentes. Por tanto, se propone fortalecer a través de alianzas 
estratégicas la política de servicios amigables el cual debe ser coordinado a través de los siguientes actores Secretaria de Educación y salud municipal, Institución educativa Paulo VI, las escuelas para padres y la comunidad Barranquera

\section{REFERENCIAS BIBLIOGRÁFICAS}

Antonio Gómez Sanabria 2017. Consumo de alcohol y drogas en estudiantes universitarios: estudio cuantitativo y cualitativo sobre prevalencia, actitudes y motivaciones para el consumo en la Universidad de Cádiz. Servicio de Publicaciones de la Universidad de Cádiz.

Congreso de Colombia, Ley 1385 de 2010, 2010.

G. M. Rodríguez Ospina, J. A. Posada Villa and S. Cárdenas, "Violencia, psique y capital social: un aporte desde el estudio nacional de salud mental, Colombia 2003.," in Una puerta hacia la paz. Paz y salud mental en Colombia., vol. 1, Academia Nacional De Medicina, 2005, pp. 83 - 96.

Gobierno Nacional de la República de Colombia, Estudio nacional de consumo de sustancias psicoactivas en población escolar. Colombia 2011. Informe final.

GUIA DE INTERVENCIÓN MHGAP. (2010). Para los trastornos mentales, neurológicos y por uso de sustancias en el nivel de atención de la salud no especializada. Organización Mundial de la salud y Programa de acción mundial Superar las brechas en salud mental. Edición original en inglés: "mhGAP intervention guide for mental, neurological and Substance use desorders in non-specialized health senttigs" World Health Organization. ISBN 9789241548069 / ISBN 9789243548067 (Clasificación NLM: WM 140). alcohol de los adolescentes: Hablando de cifras y datos. En E. Megías (Ed.), Adolescentes ante el alcohol. Barcelona: Fundació La Caixa.

Londoño Pérez Constanza 2017. Pactos por la vida: una experiencia de prevención basada en la evidencia: aproximación crítica. Universidad Católica de Colombia. http://ebookcentral. proquest.com/lib/bibliouniguajirasp/detail. action?doc $I D=4946334$.

Ministerio de Sanidad y Consumo; Secretaría General de Sanidad. "Informe sobre alcohol. Comisión Clínica de la Delegación del Gobierno para el Plan Nacional sobre Drogas.," Ministerio de Sanidad y Consumo; Secretaría General de Sanidad. Madrid, 2007.

Miranda, Martín, Miguel Angel 2012. Estrategias educativas dirigidas a desarrollar en los adolescentes y jóvenes una cultura de la salud antialcohólica desde la vía extracurricular. B Universidad de Camagüey

Moñino García 2012. Factores sociales relacionados con el consumo de alcohol en adolescentes de la región de Murcia.

Pontificia Universidad Javeriana, Estimación de la carga de enfermedad para Colombia, 2010, Bogotá, D.C.: Pontificia Universidad Javeriana, 2014. 25. Organización Panamericana de la Salud., Informe situaciones regional sobre el alcohol y la salud en las américas., 2015. Washington, D.C.: OPS. 26.

Sanabria, P. A., González-Quevedo, L. A. y Urrego, D. Z. (2007). Estilos de vida saludable en profesionales de la salud colombianos: Estudio exploratorio. Revista Med, 15, 207-217.

Valdez González, 2010. El consumo de alcohol en estudiantes del distrito federal y su relación con la autoestima y la percepción de riesgo.

La espada, T. y Elzo, J. (2007a). Consumo de 